Internat. J. Math. \& Math. Sci.

Vol. 23, No. 4 (2000) 261-270

S0161171200001010

(C) Hindawi Publishing Corp.

\title{
STABILITY OF THE POSITIVE STEADY-STATE SOLUTIONS OF SYSTEMS OF NONLINEAR VOLTERRA DIFFERENCE EQUATIONS OF POPULATION MODELS WITH DIFFUSION AND INFINITE DELAY
}

\author{
B. SHI
}

(Received 19 June 1998)

\begin{abstract}
An open problem given by Kocic and Ladas in 1993 is generalized and considered. A sufficient condition is obtained for each solution to tend to the positive steady-state solution of the systems of nonlinear Volterra difference equations of population models with diffusion and infinite delays by using the method of lower and upper solutions and monotone iterative techniques.
\end{abstract}

Keywords and phrases. Nonlinear Volterra difference equations, diffusion, infinite delays, positive steady-state solutions, methods of lower and upper solutions, monotone iterative techniques.

2000 Mathematics Subject Classification. Primary 39A10, 39A12.

1. Introduction. We consider the $r$-dimensional Euclidean space $\mathbb{R}^{r}$. For $\mathbf{x}=\left(x_{1}, \ldots\right.$, $\left.x_{r}\right)^{T} \in \mathbb{R}^{r}$, we define its norm $\|\mathbf{x}\|=\max _{i \in I}\left|x_{i}\right|$, where $I=\{1, \ldots, r\}$. In $\mathbb{R}^{r}$, we introduce a cone $P=\left\{\mathbf{x}: x_{i} \geq 0, i \in I\right\}$. Then it is a solid cone in $\mathbb{R}^{r}$. It is easy to show that $P$ is normal, regular, minimal, strong minimal and regenerated (see Amann [3]). For two elements $\mathbf{x}$ and $\mathbf{y}=\left(y_{1}, \ldots, y_{r}\right)^{T}$ in $P$, we introduce a partial ordering $\leq$ such that $\mathbf{x}<($ or $=) \mathbf{y}$ if and only if $x_{i}<($ or $=) y_{i}$ for $i \in I$ and $\mathbf{x} \leq \mathbf{y}$ means that $x_{i} \leq y_{i}$ for $i \in I$. So, $\left(\mathbb{R}^{r}, \leq\right)$ becomes a partial ordered Banach space. In $\mathbb{R}^{r}$, we also define an operation of multiplication $\otimes$ by $\mathbf{x} \otimes \mathbf{y}=\left(x_{1} y_{1}, \ldots, x_{r} y_{r}\right)^{T}$. In this way, $\left(\mathbb{R}^{r},+, \otimes\right)$ is a partially ordered commutative ring by installing both this operation $\otimes$ and the ordinary addition + with the zero element $\mathbf{0}=(0, \ldots, 0)^{T}$ and the unit element $\mathbf{u}=(1, \ldots, 1)^{T}$. Define an ordered interval $[\cdot, \cdot]$ in $\mathbb{R}^{r}$ by $[\mathbf{x}, \mathbf{y}]=\left\{\mathbf{z} \in \mathbb{R}^{r}: \mathbf{x} \leq \mathbf{z} \leq \mathbf{y}\right\}$.

In the $r \times r$-dimensional matrix space $\mathbb{R}^{r \times r}$, we also introduce a partial ordering $\leq$. If $X=\left(x_{i j}\right)_{r \times r}$ and $Y=\left(y_{i j}\right)_{r \times r}$ are two elements in $\mathbb{R}^{r \times r}$, then define that $X<($ or $=) Y$ if and only if $x_{i j}<($ or $=) y_{i j}$ for $i, j \in I$ and $X \leq Y$ means that $x_{i j} \leq y_{i j}$ for $i, j \in I$. Therefore, $\mathbb{R}^{r \times r}$ also becomes a partially ordered Banach space.

Consider the following systems of nonlinear Volterra difference equations of population model with diffusion and infinite delays:

$$
\Delta_{2} \mathbf{u}_{m, n}=A \Delta_{1}^{2} \mathbf{u}_{m-1, n+1}+\mathbf{u}_{m, n} \otimes\left(\mathbf{b}-C \mathbf{u}_{m, n}-\sum_{i=0}^{\infty} D_{i} \mathbf{u}_{m, n-i}\right)
$$

for $(m, n) \in \Omega \times Z^{+}(0):=\left\{1, \ldots, M_{1}\right\} \times \cdots \times\left\{1, \ldots, M_{s}\right\} \times\{0,1, \ldots\}$, where $\Delta_{1}$ and $\Delta_{2}$ are forward partial difference operators, $\Delta_{1}^{2}$ is a discrete Laplacian operator (see 
[7, 14, 15]), $A, C>(0)_{r \times r}$ are diagonal matrices, $\mathbf{b} \in \mathbb{R}^{r}$ and $\mathbf{b}>\mathbf{0}, \mathbf{u} ., \cdot \in \mathbb{R}^{r}$ is a double vector sequence (only in form), $D_{0}=(0)_{r \times r}$ and $D_{i} \in \mathbb{R}^{r \times r}$ for $i \in Z^{+}(0)$.

Together with (1.1), we consider the homogeneous Neumann boundary condition

$$
\Delta_{N} \mathbf{u}_{m-1, n+1}=\mathbf{0} \quad \text { for }(m, n) \in \partial \Omega \times Z^{+}(0)
$$

and the initial condition

$$
\mathbf{u}_{m, j}=\phi_{m, j} \text { for }(m, n) \in \Omega \times Z^{-}(0):=\Omega \times\{\ldots,-1,0\},
$$

where $\Delta_{N}$ is the normal difference, $\partial \Omega$ is the boundary of $\Omega$ (see [15]) and $\phi_{m, j} \in P$ for $(m, n) \in \Omega \times Z^{-}(0)$.

By a solution, we mean a double vector sequence (in form) $\left\{\mathbf{u}_{m, n}\right\}$, which is defined on $(m, n) \in \Omega \times Z:=\Omega \times Z^{+}(0) \cup Z^{-}(0)$, satisfies (1.1), (1.2), and (1.3), respectively, when $(m, n) \in \Omega \times Z^{+}(0),(m, n) \in \partial \Omega \times Z^{+}(0)$, and $(m, j) \in \Omega \times Z^{-}(0)$.

For any given initial and boundary condition (1.2) and (1.3), we can show that the initial and boundary value problem (1.1), (1.2), and (1.3) have a unique solution (see [16]).

We suppose that

$$
\begin{gathered}
\sum_{i=0}^{\infty}\left|D_{i}\right|=D<\infty, \\
0<\|\phi\|=\sup _{(m, j) \in \Omega \times Z^{-}(0)} \phi_{m, j}<\infty .
\end{gathered}
$$

We write throughout this paper that

$$
D_{n}=\sum_{i=0}^{n}\left|D_{i}\right|, \quad \delta_{n}=\sum_{i=0}^{n} D_{i}, \quad D_{n}^{ \pm}=\frac{D_{n} \pm \delta_{n}}{2} \quad \text { for } n \in Z^{+}(0) .
$$

Then $D_{n}, D_{n}^{ \pm}$are all nonnegative, nondecreasing and bounded above by $D$.

Since $P$ is regular, we can let $D^{ \pm}=\lim _{n \rightarrow \infty} D_{n}^{ \pm}$. It is easy to see that

$$
D_{n}^{+}+D_{n}^{-}=D_{n}, \quad D_{n}^{+}-D_{n}^{-}=\delta_{n}, \quad D^{+}+D^{-}=D, \quad D^{+}-D^{-}=\delta=\sum_{i=0}^{\infty} D_{i} .
$$

Assume that

$$
C \mathbf{u}>D^{-} \mathbf{u}
$$

From Berman and Plemmons [4] or Siljak [18], we know that $C-D^{-}$is a nonsingular and inverse-positive Metzlerian matrix, i.e., $C-D^{-}$is invertible and $\operatorname{det}\left(C-D^{-}\right)^{-1}>0$. Then $\left(C-D^{-}\right)^{-1} \mathbf{b}>0$. Since $C+\delta>C-D^{-}$, we know, from Metzlerian matrix theory, that $C+\delta$ is invertible and $\operatorname{det}(C+\delta)^{-1}>0$.

In addition, we let

$$
\mathbf{b}-D^{+}\left(C-D^{-}\right)^{-1} \mathbf{b}>\mathbf{0}
$$

and

$$
\mathbf{p}=\max \left\{\left(C-D^{-}\right)^{-1} \mathbf{b},\|\phi\|\right\}
$$


It is obvious that the nonlinear Volterra difference equation of population model

$$
\Delta x_{n}=x_{n}\left(b-c x_{n}-\sum_{i=0}^{\infty} d_{i} x_{n-i}\right) \text { for } n \in Z^{+}(0)
$$

is a special case when $r=1$ and without diffusion, where $\Delta$ is the forward difference operator (cf. [2, 7]).

In Kocic and Ladas [10], the following open problem was given.

Open problem. Obtain stability and oscillation results for (1.10).

Recently, many mathematicians have approached some problems of (1.10). See, e.g., Karakostas, Philos and Sficas [6], Kiventidis [8], Kocic and Ladas [9, 10], Kuruklis and Ladas [11], and Rodrigues [12], etc. In [14, 15, 16, 17], some problems for the nonlinear or linear Volterra difference equation of population models are considered.

It is easy to show that (1.1) has only two steady-state solutions $\mathbf{u}_{m, n} \equiv \mathbf{0}$ and $\mathbf{u}_{m, n} \equiv$ $(C+\delta)^{-1} \mathbf{b}$. The purpose of this paper is to give a sufficient condition for each solution of (1.1) to tend to the positive steady-state solution $\mathbf{u}_{m, n} \equiv(C+\delta)^{-1} \mathbf{b}$ of (1.1) by using the method of lower and upper solutions and monotone iterative techniques (cf. $[1,13])$.

\section{Some lemmas}

LEMMA 2.1. Let (1.4), (1.7), (1.8), and (1.9) hold. Suppose that $\left\{\mathbf{u}_{m, n}\right\}$ is the unique solution of (1.1), (1.2), and (1.3). Then,

$$
\mathbf{u}_{m, n} \in[\mathbf{0 , p}] \text { for }(m, n) \in \Omega \times Z^{+}(0) .
$$

Proof. Consider the initial and boundary value problems

$$
\begin{gathered}
\Delta_{2} \mathbf{v}_{m, n} \leq A \Delta_{1}^{2} \mathbf{v}_{m-1, n+1}+\mathbf{v}_{m, n} \otimes\left(\mathbf{b}-C \mathbf{v}_{m, n}-\sum_{i=0}^{\infty} D_{i} \mathbf{v}_{m, n-i}\right) \quad \text { for }(m, n) \in \Omega \times Z^{+}(0), \\
\Delta_{N} \mathbf{v}_{m-1, n+1}=\mathbf{0} \leq \mathbf{0} \quad \text { for }(m, n) \in \partial \Omega \times Z^{+}(0), \\
\mathbf{v}_{m, j}=\mathbf{0} \leq \phi_{m, j} \quad \text { for }(m, n) \in \Omega \times Z^{-}(0)
\end{gathered}
$$

and

$$
\begin{gathered}
\Delta_{2} \mathbf{w}_{m, n} \geq A \Delta_{1}^{2} \mathbf{w}_{m-1, n+1}+\mathbf{w}_{m, n} \otimes\left(\mathbf{b}-C \mathbf{w}_{m, n}-\sum_{i=0}^{\infty} D_{i} \mathbf{w}_{m, n-i}\right) \text { for }(m, n) \in \Omega \times Z^{+}(0), \\
\Delta_{N} \mathbf{w}_{m-1, n+1}=\mathbf{0} \geq \mathbf{0} \quad \text { for }(m, n) \in \partial \Omega \times Z^{+}(0), \\
\mathbf{w}_{m, j}=\mathbf{p} \geq \phi_{m, j} \quad \text { for }(m, n) \in \Omega \times Z^{-}(0) .
\end{gathered}
$$

Since

$$
\mathbf{b}-(C+\delta) \mathbf{p} \leq \mathbf{b}-C \mathbf{p}+D^{-} \mathbf{p}=\mathbf{b}-\left(C-D^{-}\right) \mathbf{p} \leq \mathbf{0},
$$

it is easy to see that $\mathbf{v} \equiv \mathbf{0}$ and $\mathbf{w}_{m, n} \equiv \mathbf{p}$ are, respectively, solutions of (2.2) and (2.3), i.e., a pair of lower and upper solutions of (1.1), (1.2), and (1.3). Therefore, (2.1) holds. This completes the proof. 
LEMMA 2.2. Let (1.4), (1.7), (1.8), and (1.9) hold. Suppose that $\left\{p_{n}^{(1)}\right\}$ is the unique solution of the Cauchy problem

$$
\begin{gathered}
\Delta \mathbf{p}_{n}^{(1)}=\mathbf{p}_{n}^{(1)} \otimes\left(\mathbf{b}-C \mathbf{p}_{n}^{(1)}+D^{-} \mathbf{p}\right) \quad \text { for } n \in Z^{+}(0), \\
\mathbf{p}_{j}^{(1)}=\mathbf{p} \quad \text { for } j \in Z^{-}(0) .
\end{gathered}
$$

Then $\left\{\mathbf{p}_{n}^{(1)}\right\}$ is nonincreasing and

$$
\mathbf{p}_{n}^{(1)} \in\left[C^{-1}\left(\mathbf{b}+D^{-} \mathbf{p}\right), \mathbf{p}\right] \text { for } n \in Z^{+}(0) .
$$

Proof. Consider the Cauchy problems

$$
\begin{gathered}
\Delta \mathbf{v}_{n}^{(1)} \leq \mathbf{v}_{n}^{(1)} \otimes\left(\mathbf{b}-C \mathbf{v}_{n}^{(1)}+D^{-} \mathbf{p}\right) \quad \text { for } n \in Z^{+}(0), \\
\mathbf{v}_{j}^{(1)}=C^{-1}\left(\mathbf{b}+D^{-} \mathbf{p}\right) \leq \mathbf{p} \quad \text { for } j \in Z^{-}(0),
\end{gathered}
$$

and

$$
\begin{gathered}
\Delta \mathbf{w}_{n}^{(1)} \geq \mathbf{w}_{n}^{(1)} \otimes\left(\mathbf{b}-C \mathbf{w}_{n}^{(1)}+D^{-} \mathbf{p}\right) \quad \text { for } n \in Z^{+}(0), \\
\mathbf{w}_{j}^{(1)}=\mathbf{p} \geq \mathbf{p} \quad \text { for } j \in Z^{-}(0) .
\end{gathered}
$$

It is easy to see that $\mathbf{v}_{n}^{(1)} \equiv C^{-1}\left(\mathbf{b}+D^{-} \mathbf{p}\right)$ and $\mathbf{w}_{n}^{(1)} \equiv \mathbf{p}$ are, respectively, solutions of (2.7) and (2.8), i.e., a pair of lower and upper solutions of (2.5). So, (2.6) holds.

By (2.6), we have that $\Delta \mathbf{p}_{n}^{(1)} \leq \mathbf{0}$. Hence, $\left\{\mathbf{p}_{n}^{(1)}\right\}$ is nonincreasing. The proof is thus complete.

LEMMA 2.3. Let (1.4), (1.7), (1.8), and (1.9) hold. Suppose that $\left\{\mathbf{u}_{m, n}\right\}$ and $\left\{\mathbf{p}_{n}^{(1)}\right\}$ are, respectively, the unique solutions of (1.1), (1.2), (1.3), and (2.5). Then

$$
\mathbf{u}_{m, n} \in\left[\mathbf{0}, \mathbf{p}_{n}^{(1)}\right] \quad \text { for }(m, n) \in \Omega \times Z^{+}(0) .
$$

Proof. Let $J^{ \pm}$satisfy that $J^{+} \cup J^{-}=Z^{+}(0)$ and $J^{+} \cap J^{-}=\varnothing$, the empty set, and be such that $D_{i} \geq(0)_{r \times r}$ for $i \in J^{+}$and $D_{i}<(0)_{r \times r}$ for $i \in J^{-}$. Write $\delta^{ \pm}=\sum_{i \in J^{ \pm}} D_{i}$. Then we must have $\delta^{+}=D^{+}$and $-\delta^{-}=D^{-}$. Hence, using (2.6) we have,

$$
\begin{gathered}
-\sum_{i=0}^{\infty} D_{i} \mathbf{p}_{n-i}^{(1)}=-\sum_{i \in J^{+}} D_{i} \mathbf{p}_{n-i}^{(1)}-\sum_{i \in J^{-}} D_{i} \mathbf{p}_{n-i}^{(1)} \leq-\sum_{i \in J^{-}} D_{i} \mathbf{p}_{n-i}^{(1)} \leq-\delta^{-} \mathbf{p}=D^{-} \mathbf{p}, \\
\mathbf{b}-C \mathbf{p}_{n}^{(1)}-\sum_{i=0}^{\infty} D_{i} \mathbf{p}_{n-i}^{(1)} \leq \mathbf{b}-C \mathbf{p}_{n}^{(1)}+D^{-} \mathbf{p} \quad \text { for } n \in Z^{+}(0) .
\end{gathered}
$$

Therefore, $\mathbf{w}_{m, n} \equiv \mathbf{p}_{n}^{(1)}$ is a solution of (2.3) and (2.9) holds. Thus, the proof is complete.

For the regularity of $P$, we can let $\mathbf{p}^{(1)}=\lim _{n \rightarrow \infty} \mathbf{p}_{n}^{(1)}$. By virtue of (2.5), we can obtain $\mathbf{p}^{(1)}=C^{-1}\left(\mathbf{b}+D^{-} \mathbf{p}\right)$. It follows that

$$
\limsup _{n \rightarrow \infty} \max _{m \in \Omega} \mathbf{u}_{m, n} \leq \mathbf{p}^{(1)} .
$$


So, for any $\epsilon=(\epsilon, \ldots, \epsilon)^{T}>\mathbf{0}$, there exist an $n_{1}>0$ and an $n_{2}>n_{1}$ such that

$$
\mathbf{u}_{m, n}<\mathbf{p}^{(1)}+\epsilon \text { for } n \in Z^{+}\left(n_{1}\right)
$$

and

$$
(0)_{r \times r} \leq D^{-}-D_{n-n_{1}-1}^{-}<(\epsilon)_{r \times r} \text { for } n \in Z^{+}\left(n_{2}\right) .
$$

LEMMA 2.4. Let (1.4), (1.7), (1.8), and (1.9) hold. Suppose that $\left\{p_{n}^{(2)}\right\}$ is the unique solution of the Cauchy problem

$$
\begin{gathered}
\Delta \mathbf{p}_{n}^{(2)}=\mathbf{p}_{n}^{(2)} \otimes\left(\mathbf{b}-C \mathbf{p}_{n}^{(2)}+D^{-}\left(\mathbf{p}_{1}+\epsilon\right)+\epsilon \otimes \mathbf{p}\right) \quad \text { for } n \in Z^{+}\left(n_{2}\right), \\
\mathbf{p}_{j}^{(2)}=\mathbf{p}_{1}+\epsilon \quad \text { for } j \in Z^{-}\left(n_{2}\right) .
\end{gathered}
$$

Then $\mathbf{p}_{n}^{(2)}$ is nonincreasing and

$$
\mathbf{u}_{m, n} \in\left[\mathbf{0}, \mathbf{p}_{n}^{(2)}\right] \quad \text { for }(m, n) \in \Omega \times Z^{+}\left(n_{2}\right) .
$$

Proof. If (2.15) is not true, then there exist an $m_{3} \in \Omega$ and an $n_{3}>n_{2}$ such that $\mathbf{u}_{m, n} \leq \mathbf{p}_{n}^{(2)}$ for $n_{2} \leq n<n_{3}$ and $m \in \Omega$ and $\mathbf{u}_{m_{3}, n_{3}}>\mathbf{p}_{n}^{(2)}$.

Let $\mathbf{x}_{m, n}=\mathbf{u}_{m, n}-\mathbf{p}_{n}^{(2)}$. Then $\mathbf{x}_{m, n} \leq \mathbf{0}$ for $n_{2} \leq n<n_{3}$ and $m \in \Omega$ and

$$
\mathbf{x}_{m_{3}, n_{3}}>\mathbf{0} \text {. }
$$

We can derive, from (2.14),

$$
A \Delta_{1}^{2} \mathbf{x}_{m-1, n+1}-\Delta_{2} \mathbf{x}_{m, n}+\mathbf{y}_{m, n} \otimes \mathbf{x}_{m, n}=\mathbf{z}_{m, n} \text { for }(m, n) \in \Omega \times Z^{+}\left(n_{2}\right),
$$

where

$$
\begin{gathered}
\mathbf{y}_{m, n}=\mathbf{b}-C \mathbf{u}_{m, n}-\sum_{i=0}^{\infty} D_{i} \mathbf{u}_{m, n-i}-C \mathbf{p}_{n}^{(2)} \quad \text { for }(m, n) \in \Omega \times Z^{+}\left(n_{2}\right), \\
\mathbf{z}_{m, n}=\mathbf{p}_{n}^{(2)} \otimes\left(D^{-}\left(\mathbf{p}_{1}+\epsilon\right)+\sum_{i=0}^{\infty} D_{i} \mathbf{u}_{m, n-i}+\epsilon \otimes \mathbf{p}\right) \quad \text { for }(m, n) \in \Omega \times Z^{+}\left(n_{2}\right) .
\end{gathered}
$$

It is easy to show that $\mathbf{y}_{m, n}$ is bounded. We can see in the following that $\mathbf{z}_{m, n} \geq \mathbf{0}$. Indeed, from (2.12) and (2.13), we have

$$
\begin{aligned}
-\sum_{i=0}^{\infty} D_{i} \mathbf{u}_{m, n-i} & =-\sum_{i=0}^{\infty}\left(\Delta \delta_{i-1}\right) \mathbf{u}_{m, n-i} \\
& =-\sum_{i=0}^{\infty}\left(\Delta D_{i-1}^{+}\right) \mathbf{u}_{m, n-i}+\sum_{i=0}^{\infty}\left(\Delta D_{i-1}^{-}\right) \mathbf{u}_{m, n-i} \\
& \leq \sum_{i=0}^{n-n_{1}-1}\left(\Delta D_{i-1}^{-}\right) \mathbf{u}_{m, n-i}+\sum_{i=n-n_{1}}^{\infty}\left(\Delta D_{i-1}^{-}\right) \mathbf{u}_{m, n-i} \\
& \leq D_{n-n_{1}-1}^{-}\left(\mathbf{p}_{1}+\epsilon\right)+\left(D^{-}-D_{n-n_{1}-1}^{-}\right) \mathbf{p} \\
& \leq D^{-}\left(\mathbf{p}_{1}+\epsilon\right)+(\epsilon)_{r \times r} \mathbf{p} .
\end{aligned}
$$

So, $\mathbf{z}_{m, n} \geq \mathbf{0}$ for $(m, n) \in \Omega \times Z^{+}\left(n_{2}\right)$ from (2.18) and (2.19). It follows, from (2.17), that

$$
\Delta_{2} \mathbf{x}_{m, n} \leq A \Delta_{1}^{2} \mathbf{x}_{m-1, n+1}+\mathbf{y}_{m, n} \otimes \mathbf{x}_{m, n} .
$$


Consider the initial and boundary problems

$$
\begin{gathered}
\Delta_{2} \mathbf{v}_{m, n}=A \Delta_{1}^{2} \mathbf{v}_{m-1, n+1}+\mathbf{y}_{m, n} \otimes \mathbf{v}_{m, n} \text { for }(m, n) \in \Omega \times Z^{+}\left(n_{2}\right), \\
\Delta_{N} \mathbf{v}_{m-1, n+1}=\mathbf{0} \text { for }(m, n) \in \partial \Omega \times Z^{+}\left(n_{2}\right), \\
\mathbf{v}_{m, n_{2}}=\mathbf{0} \text { for } m \in \Omega
\end{gathered}
$$

and

$$
\begin{gathered}
\Delta_{2} \mathbf{x}_{m, n} \leq A \Delta_{1}^{2} \mathbf{x}_{m-1, n+1}+\mathbf{y}_{m, n} \otimes \mathbf{x}_{m, n} \text { for }(m, n) \in \Omega \times Z^{+}\left(n_{2}\right), \\
\Delta_{N} \mathbf{x}_{m-1, n+1} \leq \mathbf{0} \text { for }(m, n) \in \partial \Omega \times Z^{+}\left(n_{2}\right), \\
\mathbf{x}_{m, n_{2}} \leq \mathbf{0} \text { for } m \in \Omega .
\end{gathered}
$$

Obviously, $\mathbf{v}_{m, n} \equiv \mathbf{0}$ is the unique solution of (2.21). Comparing (2.21) with (2.22), we see that $\mathbf{x}_{m, n} \leq \mathbf{0}$ for $(m, n) \in \Omega \times Z^{+}\left(n_{2}\right)$. But, this contradicts (2.16). Therefore, (2.15) holds.

Similarly to the proof of Lemma 2.2, we can easily show that $p_{n}^{(2)}$ is nonincreasing, which completes the proof.

REMARK 2.5. As a matter of fact, we can directly use the maximum principle (see Cheng [5]) to obtain the contradiction.

We can obtain from (2.14) and the regularity of $P$ that

$$
\lim _{n \rightarrow \infty} \mathbf{p}_{n}^{(2)}=C^{-1}\left(\mathbf{b}+D^{-}\left(\mathbf{p}_{1}+\epsilon\right)+\epsilon \otimes \mathbf{p}\right) .
$$

Therefore,

$$
\limsup _{n \rightarrow \infty} \max _{m \in \Omega} \mathbf{u}_{m, n} \leq C^{-1}\left(\mathbf{b}+D^{-}\left(\mathbf{p}_{1}+\epsilon\right)+\epsilon \otimes \mathbf{p}\right) .
$$

Because $\epsilon$ is arbitrary, we have

$$
\limsup _{n \rightarrow \infty} \max _{m \in \Omega} \mathbf{u}_{m, n} \leq C^{-1}\left(\mathbf{b}+D^{-} \mathbf{p}_{1}\right):=\mathbf{p}_{2} .
$$

Define a sequence $\left\{\mathbf{p}_{\ell}\right\}$ as follows:

$$
\begin{gathered}
\mathbf{p}_{\ell}=C^{-1}\left(\mathbf{b}+D^{-} \mathbf{p}_{\ell-1}\right) \quad \text { for } \ell \in Z^{+}(1), \\
\mathbf{p}_{0}=\mathbf{p} .
\end{gathered}
$$

LEMMA 2.6. Let (1.4), (1.7), (1.8), and (1.9) hold. Suppose that $\left\{\mathbf{p}_{\ell}\right\}$ is defined by (2.26). Then, $\left\{\mathbf{p}_{\ell}\right\}$ is nonincreasing and

$$
\left(C-D^{-}\right)^{-1} \mathbf{b} \in\left[\mathbf{0}, \mathbf{p}_{\ell}\right] \quad \text { for } \ell \in Z^{+}(0) .
$$

Proof. We rewrite (2.26) as follows:

$$
\Delta \mathbf{p}_{\ell}=C^{-1} D^{-} \Delta \mathbf{p}_{\ell-1} \quad \text { for } \ell \in Z^{+}(1) .
$$

We know from $\Delta \mathbf{p}_{0}=\mathbf{p}_{1}-\mathbf{p} \leq \mathbf{0}$ that $\Delta \mathbf{p}_{\ell} \leq \mathbf{0}$ for all $\ell \in Z^{+}(0)$. That is, $\left\{\mathbf{p}_{\ell}\right\}$ is nonincreasing. 
Noting that $\mathbf{p} \geq\left(C-D^{-}\right)^{-1} \mathbf{b}$, we have from (2.26) and (2.6), that $C \mathbf{p}_{1}=\mathbf{b}+D^{-} \mathbf{p} \geq$ $\mathbf{b}+D^{-} \mathbf{p}_{1}$. Hence, $\mathbf{p}_{1} \geq\left(C-D^{-}\right)^{-1} \mathbf{b}$. By induction, we obtain (2.27). This completes the proof.

Because $P$ is regular, we let $\gamma=\lim _{\ell \rightarrow \infty} \mathbf{p}^{(\ell)}$. From (2.26), we have $\gamma=C^{-1}\left(\mathbf{b}+D^{-} \gamma\right)$. We can solve $\gamma=\left(C-D^{-}\right)^{-1} \mathbf{b}$.

Repeating the above procedure, we can show that

$$
\limsup _{n \rightarrow \infty} \max _{m \in \Omega} u_{m, n} \leq \gamma .
$$

From (1.8), we have that $\mathbf{b}>D^{+} \gamma$. So, we can select an $\epsilon_{0}>\mathbf{0}$ such that $\mathbf{b}>D^{+}\left(\gamma+\epsilon_{0}\right)$ $+\epsilon_{0} \otimes \mathbf{p}$.

Let $\mathbf{0}<\epsilon<\epsilon_{0}$. By (2.19), there exist an $n_{4}>n_{3}$ and an $n_{5}>n_{4}$ such that

$$
\begin{gathered}
\mathbf{u}_{m, n}<\gamma+\epsilon \text { for }(m, n) \in \Omega \times Z^{+}\left(n_{4}\right), \\
(0)_{r \times r} \leq D^{+}-D_{n-n_{4}-1}^{+}<(\epsilon)_{r \times r} \text { for } n \in Z^{+}\left(n_{5}\right) .
\end{gathered}
$$

From (1.2), Lemma 2.1, and maximum principle (see Cheng [5]), we know that $\mathbf{u}_{m, n}>\mathbf{0}$ for $(m, n) \in \Omega \times Z^{+}(0)$ and can select an $\eta>\mathbf{0}$ such that $\min _{m \in \Omega} \mathbf{u}_{m, n_{5}} \geq 2 \eta$.

Consider the Cauchy problem

$$
\begin{gathered}
\Delta \mathbf{q}_{n}=\mathbf{q}_{n} \otimes\left(\mathbf{b}-C \mathbf{q}_{n}-D^{+}(\gamma+\epsilon)-\epsilon \otimes \mathbf{p}\right) \quad \text { for } n \in Z^{+}\left(n_{5}\right), \\
\mathbf{q}_{j}=\eta \quad \text { for } j \in Z^{-}\left(n_{5}\right) .
\end{gathered}
$$

Repeating an argument similar to the above, we can obtain that $\mathbf{q}_{n}<\mathbf{u}_{m, n}$ for $(m, n) \in \Omega \times Z^{+}\left(n_{5}\right)$ and

$$
\lim _{n \rightarrow \infty} \mathbf{q}_{n}=C^{-1}\left(\mathbf{b}-D^{+}(\gamma+\epsilon)-\epsilon\right) \otimes \mathbf{p}
$$

Consequently, we have

$$
\liminf _{n \rightarrow \infty} \min _{m \in \Omega} \mathbf{u}_{m, n} \geq C^{-1}\left(\mathbf{b}-D^{+} \gamma\right)
$$

for $\epsilon>\mathbf{0}$ being arbitrary.

Define a pair of coupled sequences $\left\{\mu_{k}\right\}$ and $\left\{v_{k}\right\}$ as follows:

$$
\begin{array}{rlrl}
C \mu_{k} & =\mathbf{b}-D^{+} v_{k-1}+D^{-} \mu_{k-1} & & \text { for } k \in Z^{+}(1), \\
C v_{k} & =\mathbf{b}+D^{-} v_{k-1}-D^{+} \mu_{k-1} & & \text { for } k \in Z^{+}(1), \\
v_{0} & =\left(C-D^{-}\right)^{-1} \mathbf{b}, \quad \mu_{0}=C^{-1}\left(\mathbf{b}-D^{+} v_{0}\right) .
\end{array}
$$

LEMMA 2.7. Let (1.4), (1.7), (1.8), and (1.9) hold. Suppose that the pair of $\left\{\mu_{k}\right\}$ and $\left\{v_{k}\right\}$ is defined by (2.34). Then,

$$
\left[\mu_{0}, v_{0}\right] \supseteq\left[\mu_{1}, v_{1}\right] \supseteq \cdots \supseteq\left[\mu_{k}, v_{k}\right] \supseteq \cdots \quad \text { for } k \in Z^{+}(0)
$$

and

$$
\lim _{k \rightarrow \infty} \mu_{k}=\lim _{k \rightarrow \infty} v_{k}=(C+\delta)^{-1} \mathbf{b}
$$


Proof. Because

$$
\begin{gathered}
C \mu_{1} \geq \mathbf{b}-D^{+} v_{0}=C \mu_{0}, \quad C v_{0}=\left(C-D^{-}\right) v_{0}+D^{-} v_{0}=\mathbf{b}+D^{-} v_{0} \geq C v_{1}, \\
C v_{0} \geq\left(C-D^{-}\right) v_{0}=\mathbf{b} \geq \mathbf{b}-D^{+} v_{0}=C \mu_{0},
\end{gathered}
$$

we have

$$
\left[\mu_{0}, v_{0}\right] \supseteq\left[\mu_{1}, v_{1}\right]
$$

We can get (2.35) by induction.

By virtue of the regularity of $P$, we can let $\mu=\lim _{k \rightarrow \infty} \mu_{k}$ and $v=\lim _{k \rightarrow \infty} v_{k}$. Then we get

$$
C \mu=\mathbf{b}-D^{+} v+D^{-} \mu, \quad C v=\mathbf{b}+D^{-} v-D^{+} \mu .
$$

Subtracting the two equalities in (2.39), we obtain $C(\mu-v)=\left(D^{+}+D^{-}\right)(\mu-v)=$ $D(\mu-v)$. So, $(C-D)(\mu-v)=\mathbf{0}$.

Since $(C-D) v_{0}=\left(C-D^{+}-D^{-}\right)\left(C-D^{-}\right)^{-1} \mathbf{b}=\mathbf{b}-D^{+}\left(C-D^{-}\right)^{-1} \mathbf{b}>\mathbf{0}$ from (1.8), we have, from the properties of Metzlerian matrices, that $\operatorname{det}(C-D)^{-1}>0$. Therefore, $\mu=v$.

It follows from (2.39) that $C \mu=\mathbf{b}-D^{+} \mu+D^{-} \mu=\mathbf{b}-\delta \mu$ or $(C+\delta) \mu=\mathbf{b}$. This leads to (2.36). The proof is thus complete.

LEMMA 2.8. Let (1.4), (1.7), (1.8), and (1.9) hold. Suppose that the pair of $\left\{\mu_{k}\right\}$ and $\left\{v_{k}\right\}$ is defined by (2.34). Then,

$$
\left[\liminf _{n \rightarrow \infty} \min _{m \in \Omega} \mathbf{u}_{m, n}, \limsup _{n \rightarrow \infty} \max _{m \in \Omega} \mathbf{u}_{m, n}\right] \in\left[\mu_{k}, v_{k}\right] \quad \text { for } k \in Z^{+}(0) .
$$

Proof. From the above, (2.40) holds for $k=0$.

Take an $\epsilon_{1}>0$ such that $\epsilon_{1}<\mu_{0}$ and

$$
\mathbf{b}>D^{+}\left(v_{0}+\epsilon_{1}\right)-D^{-}\left(\mu_{0}-\epsilon_{1}\right)+2 \epsilon_{1} \otimes \mathbf{p} .
$$

For $\mathbf{0}<\epsilon<\epsilon_{1}$, there exist an $n_{6}>n_{5}$ and an $n_{7}>n_{6}$ such that

$$
\begin{aligned}
& \mu_{0}-\epsilon<\mathbf{u}_{m, n}<v_{0}+\epsilon \text { for }(m, n) \in \Omega \times Z^{+}\left(n_{6}\right), \\
& (0)_{r \times r} \leq D-D_{n-n_{6}-1}<(\epsilon)_{r \times r} \text { for } n \in Z^{+}\left(n_{7}\right) .
\end{aligned}
$$

Now, we consider the Cauchy problems

$$
\begin{gathered}
\Delta \overline{\mathbf{p}}_{n}=\overline{\mathbf{p}}_{n} \otimes\left(\mathbf{b}-C \overline{\mathbf{p}}_{n}+D^{-}\left(\nu_{0}+\epsilon\right)-D^{+}\left(\mu_{0}-\epsilon\right)+2 \epsilon \otimes \mathbf{p}\right) \text { for } n \in Z^{+}\left(n_{7}\right), \\
\overline{\mathbf{p}}_{j}=v_{0}+\epsilon \text { for } j \in Z^{-}\left(n_{7}\right),
\end{gathered}
$$

and

$$
\begin{gathered}
\Delta \overline{\mathbf{q}}_{n}=\overline{\mathbf{q}}_{n} \otimes\left(\mathbf{b}-C \overline{\mathbf{q}}_{n}-D^{+}\left(v_{0}+\epsilon\right)+D^{-}\left(\mu_{0}-\epsilon\right)-2 \epsilon \otimes \mathbf{p}\right) \text { for } n \in Z^{+}\left(n_{7}\right), \\
\overline{\mathbf{q}}_{j}=\mu_{0}-\epsilon \text { for } j \in Z^{-}\left(n_{7}\right) .
\end{gathered}
$$


Similarly to the above argument, we can obtain

$$
\begin{aligned}
\overline{\mathbf{q}}_{n} & <\mathbf{u}_{m, n}<\overline{\mathbf{p}}_{n} \quad \text { for }(m, n) \in \Omega \times Z^{+}\left(n_{7}\right), \\
\lim _{n \rightarrow \infty} \overline{\mathbf{p}}_{n} & =C^{-1}\left(\mathbf{b}+D^{-}\left(v_{0}+\epsilon\right)-D^{+}\left(\mu_{0}-\epsilon\right)+2 \epsilon \otimes \mathbf{p}\right), \\
\lim _{n \rightarrow \infty} \overline{\mathbf{q}}_{n} & =C^{-1}\left(\mathbf{b}-D^{+}\left(\nu_{0}+\epsilon\right)+D^{-}\left(\mu_{0}-\epsilon\right)-2 \epsilon \otimes \mathbf{p}\right) .
\end{aligned}
$$

Letting $\epsilon \rightarrow \mathbf{0}$, we see that (2.40) holds for $k=1$.

Again, by repeating the above process, we have that (2.40) holds.

3. Main results and remarks. Using the seven lemmas in Section 2, together with the property that $P$ is normal, we get the following main result.

ThEOREM 3.1. Let (1.4), (1.7), (1.8), and (1.9) hold. Assume that $\left\{\mathbf{u}_{m, n}\right\}$ is the unique solution of (1.1), (1.2), and (1.3). Then

$$
\lim _{\substack{n \rightarrow \infty \\ m \in \Omega}} \mathbf{u}_{m, n}=(C+\delta)^{-1} \mathbf{b}
$$

For (1.10), we consider the initial condition

$$
x_{j}=\phi_{j} \geq 0 \quad \text { for } j \in Z^{-}(0) .
$$

Then, we have the following.

Corollory 3.2. Assume that $c>\sum_{i=0}^{\infty}\left|d_{i}\right|, 0<\|\phi\|=\sup _{j \in Z^{-}(0)} \phi_{j}<\infty$ and $\left\{x_{n}\right\}$ is the unique solution of (1.10) with (3.2). Then

$$
\lim _{n \rightarrow \infty} x_{n}=\frac{b}{c+\sum_{i=0}^{\infty} d_{i}} .
$$

REMARK 3.3. It is well known that (1.1) describes the growth of $r$-species alive in $\Omega$, that the densities of the $r$-populations at place $m$ and time $n$ is $\mathbf{u}_{m, n}$, and that the summation represents the effects of the past history on the present growth rate in mathematical ecology. Therefore, we can only consider the case $\|\phi\|>0$. If this is not the case, these species do not exist. The condition $\|\phi\|<\infty$ means that the densities of these species should be finite in practice. Relation (3.1) means that the growth of these species goes to an equilibrium state under ordinary conditions. Equation (1.10) is the case that we do not consider the places and diffusion.

ACKNOWLEDGEMENT. This project is supported by the National Natural Science Foundation of China (\# 19601016).

\section{REFERENCES}

[1] R. P. Agarwal, Comparison results for multidimensional difference equations, J. Math. Anal. Appl. 135 (1988), no. 2, 476-487. MR 89k:39001. Zbl 661.39004.

[2] _ _ Difference Equations and Inequalities, Marcel Dekker Inc., New York, 1992. MR 92m:39002. Zbl 925.39001.

[3] H. Amann, Fixed point equations and nonlinear eigenvalue problems in ordered Banach spaces, SIAM Rev. 18 (1976), no. 4, 620-709. MR 54\#3519. Zbl 345.47044. 
[4] A. Berman and R. J. Plemmons, Nonnegative Matrices in the Mathematical Sciences, Academic Press [Harcourt Brace Jovanovich Publishers], New York, 1979. MR 82b:15013. Zbl 484.15016.

[5] S. S. Cheng, Maximum Principles for Solutions of Second Order Partial Difference Inequalities, Symp. on Functional Anal. and Appl. (Taiwan), Tsing Hua univ., 1980, pp. 395401.

[6] G. Karakostas, C. G. Philos, and Y. G. Sficas, The dynamics of some discrete population models, Nonlinear Anal. 17 (1991), no. 11, 1069-1084. MR 92j:39004. Zbl 760.92019.

[7] W. G. Kelley and A. C. Peterson, Difference Equations, Academic Press Inc., Boston, MA, 1991. MR 93f:39002. Zbl 733.39001.

[8] T. Kiventidis, Positive solutions of integrodifferential and difference equations with unbounded delay, Glasgow Math. J. 35 (1993), no. 1, 105-113. MR 93m:34112. Zbl 777.45003.

[9] V. L. Kocić and G. Ladas, Global attractivity in nonlinear delay difference equations, Proc. Amer. Math. Soc. 115 (1992), no. 4, 1083-1088. MR 92j:39005. Zbl 756.39005.

[10] _ Global Behavior of Nonlinear Difference Equations of Higher Order with Applications, Kluwer Academic Publishers Group, Dordrecht, 1993. MR 94k:39005. Zbl 787.39001.

[11] S. A. Kuruklis and G. Ladas, Oscillations and global attractivity in a discrete delay logistic model, Quart. Appl. Math. 50 (1992), no. 2, 227-233. MR 93b:92013. Zbl 799.39004.

[12] I. W. Rodrigues, Oscillation and attractivity in a discrete model with quadratic nonlinearity, Appl. Anal. 47 (1992), no. 1, 45-55. MR 94b:39010. Zbl 788.39001.

[13] Q. Sheng and R. P. Agarwal, Monotone methods for higher-order partial difference equations, Comput. Math. Appl. 28 (1994), no. 1-3, 291-307. MR 95h:39005. Zbl 812.65130.

[14] B. Shi, Z. C. Wang, and J. S. Yu, Asymptotic constancy of solutions of linear parabolic Volterra difference equations, Comput. Math. Appl. 32 (1996), no. 8, 65-77. MR 99c:39008. Zbl 873.39007.

[15] _ Oscillation of nonlinear partial difference equations with delays, Comput. Math. Appl. 32 (1996), no. 12, 29-39. MR 98e:39004. Zbl 877.35131.

[16] _ Square-summable stability in parabolic Volterra difference equations, Methods Appl. Anal. 3 (1996), no. 2, 273-284. MR 98a:39010. Zbl 868.39004.

[17] Stability of parabolic Volterra integro-partial differential equations and their discrete analogues, Differential Equations Dynam. Systems 4 (1996), no. 2, 177-190. MR 99h:45020. Zbl 869.45006.

[18] Dragoslav D. Šiljak, Large-scale Dynamic Systems, North-Holland Publishing Co., New York, 1979. MR 82j:93004. Zbl 384.93002.

Shi: Department of Basic Sciences, NAVAl Aeronautical Engineering ACAdemy, YANTAI, SHANDONG 264001, CHINA 


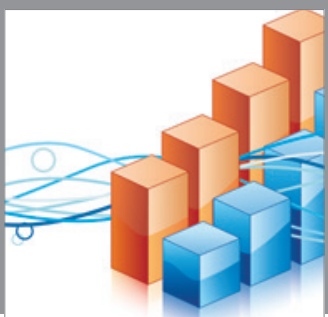

Advances in

Operations Research

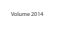

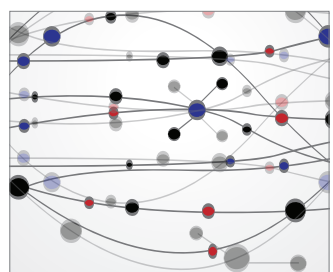

\section{The Scientific} World Journal
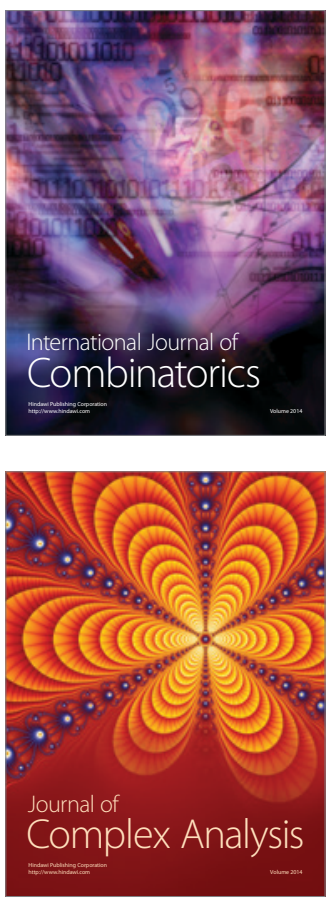

International Journal of

Mathematics and

Mathematical

Sciences
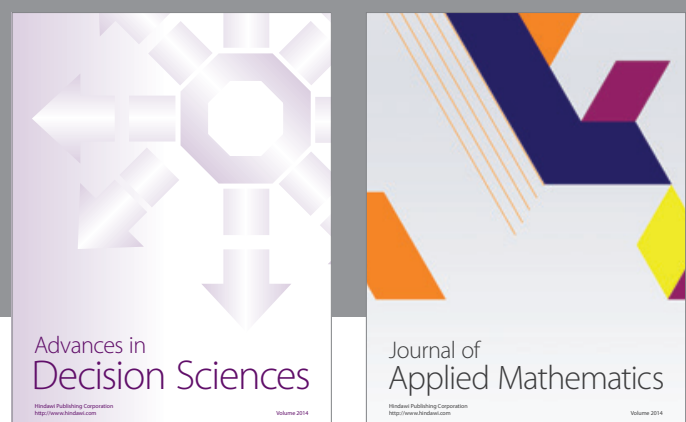

Journal of

Applied Mathematics
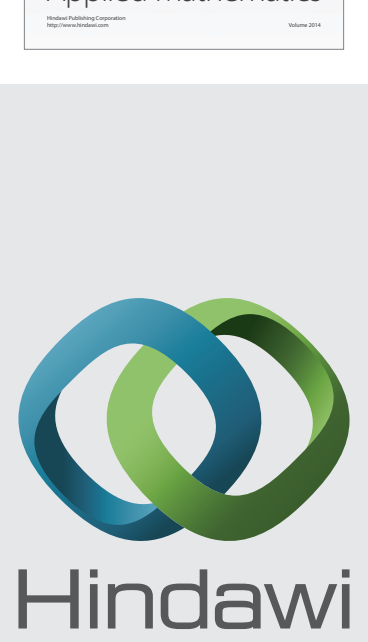

Submit your manuscripts at http://www.hindawi.com
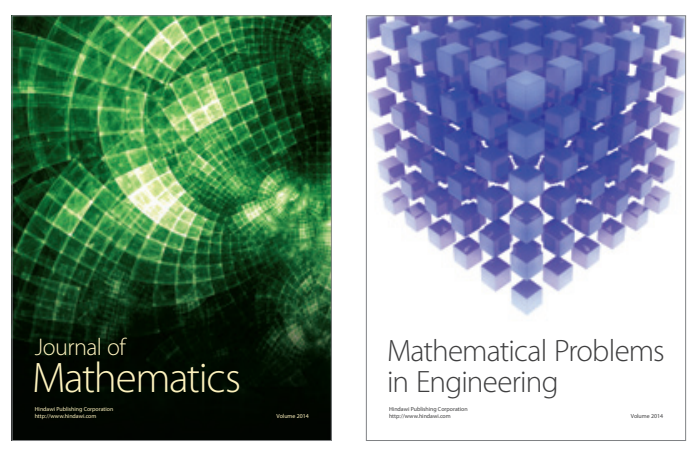

Mathematical Problems in Engineering
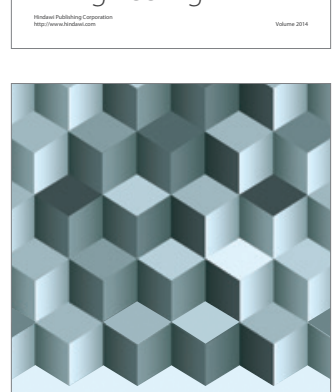

Journal of

Function Spaces
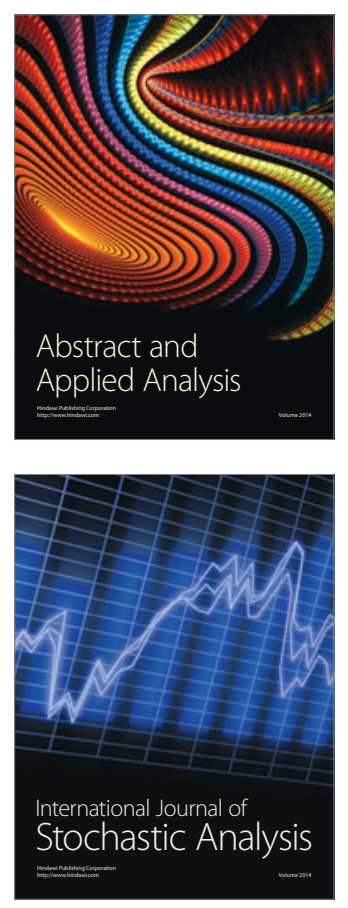

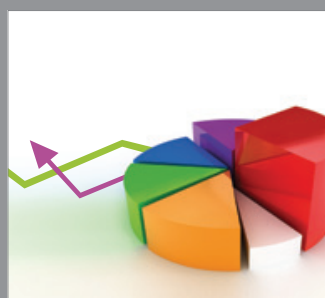

ournal of

Probability and Statistics

Promensencen
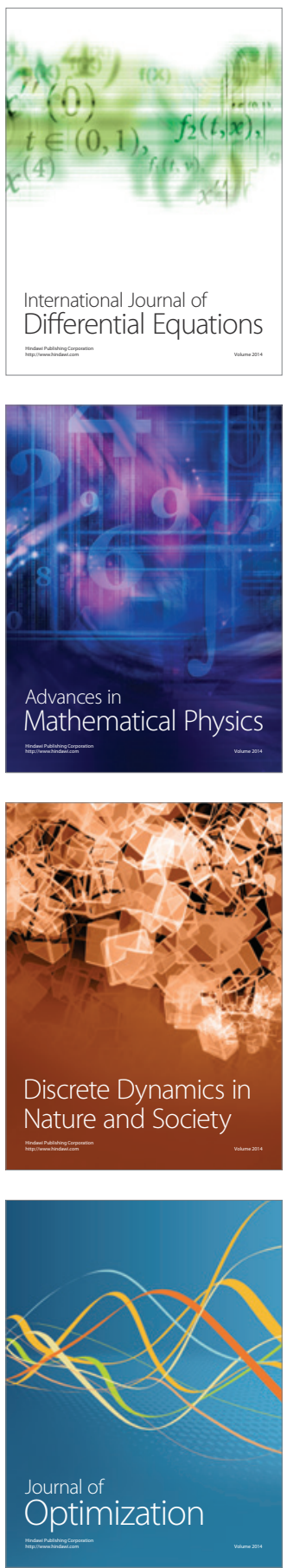